Supporting Information

\title{
Planar Orientation and Transparency of Nanoporous-crystalline Polymer Films
}

\author{
Baku Nagendra, Paola Rizzo, Christophe Daniel, Gaetano Guerra*
}

Dipartimento di Chimica e Biologia, INSTM Research Unit, Università di Salerno, Via Giovanni

Paolo II 132, 84084 Fisciano (SA), Italy

* E-mail: gguerra@unisa.it. 

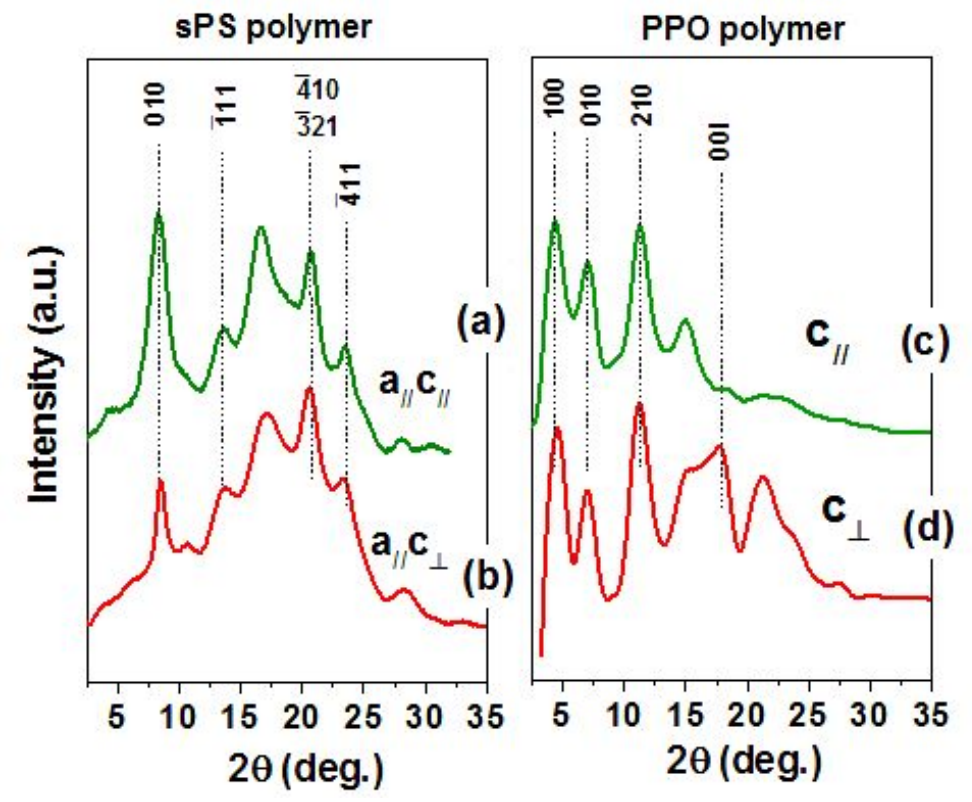

Figure S1. Radial profiles of the WAXD patterns of Figure 1 for NC sPS $(a, b)$ and NC PPO (c,d) films as obtained by guest extraction from a: (a) $\mathrm{CHCl}_{3}$ cast $\mathrm{sPS}$ film with $\mathrm{a}_{/ / \mathrm{c} / /}$ orientation; (b) TCE cast sPS film with $\mathrm{a}_{/} \mathrm{c}_{\perp}$ orientation; (c) BE cast PPO film with $\mathrm{c}_{/ /}$orientation; (d) amorphous PPO film whose crystallization was induced by BE sorption, with $\mathrm{c}_{\perp}$ orientation.

Table S1. The correlation length $(D)$ values, estimated from WAXD radial profiles (Figure S1) of 2D-WAXD EDGE patterns (Figure 1). For $D$ evaluations, 301/ $\overline{3} 21$ and 210 reflections were used for sPS and PPO, respectively. The observed kind of planar orientation is indicated in parentheses.

Films

sPS $\left(a_{/ /} c_{\|}\right)$

$\operatorname{sPS}\left(a_{\|} c_{\perp}\right)$

PPO (c

$\operatorname{PPO}\left(c_{\perp}\right)$

\section{Reflection D (nm)}

301/ $\overline{3} 21$

7.70

7.58

210

4.84

5.43 

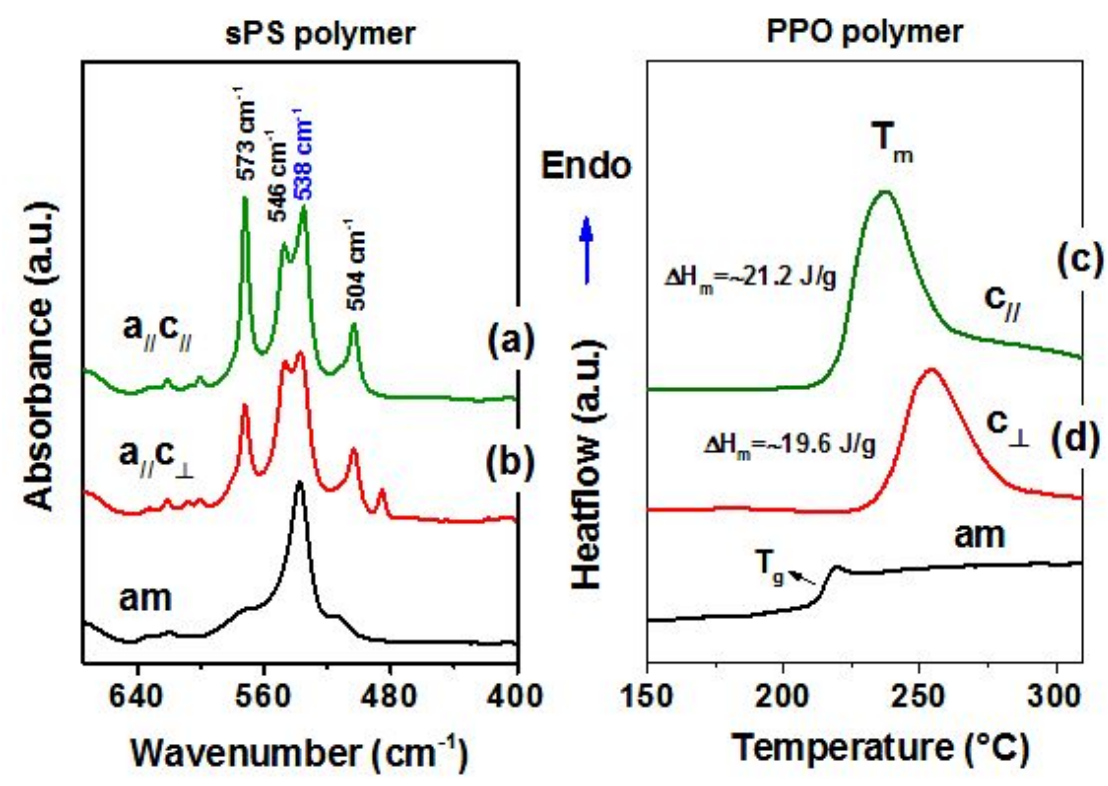

Figure S2. FTIR spectra and DSC scans of the NC sPS and NC PPO films (of Figure 1), which are used to evaluate the degree of crystallinity of the films: (a) $\mathrm{CHCl}_{3}$ cast sPS film with a//c// orientation; (b) TCE cast sPS film with $\mathrm{a} / \mathrm{c}_{\perp}$ orientation; (c) BE cast PPO film with $\mathrm{c} / /$ orientation; (d) amorphous PPO film whose crystallization was induced by BE sorption, with $\mathrm{c}_{\perp}$ orientation. For comparison, FTIR spectrum and the DSC scan of amorphous films are also shown in bottom of the Figure.
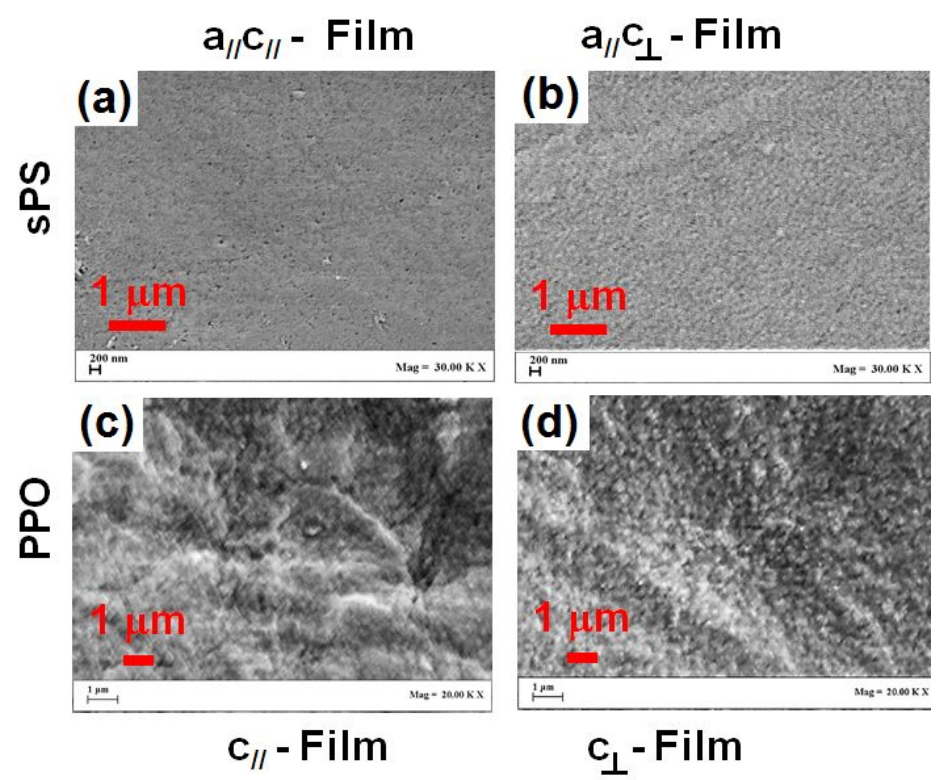

Figure S3. SEM surface morphology of NC sPS $(a, b)$ and NC PPO (c,d) films: obtained from (a) $\mathrm{CHCl}_{3}$ cast, $\mathrm{a} / / \mathrm{c}_{/} /$; (b) TCE cast, $\mathrm{a}_{/ / \mathrm{c}_{\perp}}$ orientation; (c) BE cast, $\mathrm{c}_{/ /}$; and (d) BE guest induced crystallization of amorphous $\mathrm{PPO}$ film, $\mathrm{c}_{\perp}$ orientation. 